\title{
Analysis of the Connotation of the Ambidexterity Strategic Alliance Based on a Context Ambidexterity Perspective-Taking "the Accenture-Huawei-SAP Strategic Alliance” as an Example
}

\author{
Yuquan Zuo, Deyun Xiao a, * \\ School of Economics, Wuhan University of Technology, Wuhan, Hubei, 430000, China. \\ *, a Deyunxiao@163.com
}

\begin{abstract}
In recent years, strategic alliances between companies have become commonplace. However, due to the complexity of the market and multiple conflicts between alliance partners, the development of strategic alliances is in trouble. In the current situation, one of the key of corporate work is how to have ambidexterity capabilities; another key is how to establish an effective ambidexterity strategic alliance. Therefore, whether or not ambidexterity can be achieved and the establishment of an ambidexterity strategic alliance has become the key to whether the company can obtain sustainable competitive advantage in the future. Based on the current theory of strategic alliances and ambidexterity strategy, this paper analyzes the connotation of the ambidexterity strategic alliances based on context ambidexterity from concept to dimension, and analyzes the reality of the ambidexterity strategic alliances using the "Accenture-Huawei-SAP Strategic Alliance "as an example.
\end{abstract}

Keywords: strategic alliance; ambidexterity strategy; context ambidexterity; learning ambidexterity; innovation ambidexterity.

\section{基于情境双元视角双元战略联盟的内涵分析一以 “埃森哲-华为-SAP战略联 盟”为例}

\author{
左钰泉, 肖德云 ${ }^{*}$ \\ 武汉理工大学, 经济学院, 湖北武汉, 430000 中国
}

摘 要: 近年来, 企业间的战略联盟已经成为普遍现象。但是由于市场的复杂性和联盟伙伴之 间的多重矛盾, 战略联盟的发展陷入困境。在当今形势下, 企业工作的重点之一是如何具备 双元能力; 重点之二就是如何建立有效的双元战略联盟。因此, 能否获取双元能力建立双元 战略联盟已成为未来企业能否获取持续性竞争优势的关键。本文基于目前的关于战略联盟和 双元的理论, 基于情境双元, 从概念到维度, 对双元战略联盟进行内涵分析, 以“埃森哲一 华为-SAP战略联盟”为例剖析双元战略联盟现实意义。

关键词：战略联盟；双元；情境双元；双元学习；双元创新

\section{1. 引言}

随着经济政治环境不确定性的增强和产品生命周期的日益缩短，随着生产、科技、贸易、信 息和金融全球化趋势的加快，即使是大型企业也意识到仅仅凭借自身的力量，很难从外部获 取有效知识。因此, 越来越多的企业通过组建战略联盟, 通过合作来拓宽知识基础, 以获取 知识并产生协同效应提升创新能力。实际中，企业形成的战略联盟，既包括相似性企业联盟 之间的合作，又包括产品或资源互补性企业之间的联盟合作。通过对战略联盟中企业知识、 人才、技术和管理等方面资源的整合，联盟中的企业不仅提高了抵抗外部风险能力、丰富自 身资源并且降低了获取资源的成本，而且强化了联盟在同行业或者跨行业中的整体实力。但 是，根据美国麦肯锡咨询公司的数据，在美国 800 多家公司联盟中，只有 $40 \%$ 的联盟能维持 
四年以上。2012 年，科技部 56 家国家级产业技术创新战略试点开展绩效评估后，仅 26 家联 盟优秀, 且另有四家联盟试点被取消资格。研究发现, 导致联盟失败的原因很多, 如投机主 义风险、契约风险、政府政策改变等多方面。同时，在战略联盟成员的合作中，联盟成员间 的实力并不相当、真正的目的也不相同，弱势一方想通过合作减少自己技术和管理上的劣势， 而强势一方除了对于市场份额的占有外，还隐藏对合作企业兼并或者吞并的野心，真实目的 的不同也将对联盟稳定性造成影响。

近年来, 学术界对双元能力研究的重视程度不断提高。学者们普遍的观点认为: 在内外部环 境复杂多变的情况下, 成功的组织既能有效管理现有业务, 又能主动应对未来市场变化。具 备双元能力的组织能够充分平衡和融合组织中的各种相互竞争和相互矛盾的因素。双元能力 理论解释了许多复杂的组织现象, 也被更多的用于改善战略管理、激发创新、加强组织学习 和优化组织结构设计。因此, 基于对长期绩效的追求, 为了解决战略悖论, 应对动态环境的 变化、日益激烈的市场竞争和有限的内部资源和能力间的矛盾，企业战略联盟必须具备双元 性能力。

但是, 现有的大量文献主要讨论的是组织内的双元能力, 即团队层面和组织层面, 却较少涉 及以联盟为代表的组织间层面的双元能力, 双元战略联盟的内涵还十分模糊。本文基于情境 双元的视角, 分析双元战略联盟的内涵, 研究结论可以为联盟组织如何构建有效的双元战略 联盟提供一定启示与思考。

\section{2. 文献综述}

\section{1. 战略联盟}

战略联盟最早是由 J. Hopland 和 R. Nigel 提出, 被视作企业等组织间的一种合作安排, 获 得领先竞争优势的机会。自 20 世纪 80 年代起, 全球战略联盟的数量激增, 成为企业获得竞 争优势的重要手段之一, 联盟理论研究也得以持续发展。学术界对联盟现象的分析有多种不 同的视角, 研究内容包括联盟动因、联盟治理及结构演化、联盟绩效、情境研究等诸多方面。 例如, Linsu Kim 等提出了 “组织学习” 理论以解释战略联盟, 其指出, 战略联盟是组织学 习的重要方式之一, 强调学习知识是组织竞争优势和核心竞争力的关键源泉; 但其理论其抽 象性较高, 对知识的度量难度大, 对实践的指导操作性不足, 同时也在一定程度上忽视了知 识创新。因此, 本文试图基于情境双元视角下从双元学习与双元创新的角度对双元战略联盟 进行定义。

\section{2. 双元}

自从 Duncan（1976）年第一次将双元的概念提出, 越来越多的学者对双元产生兴趣。双元视 角已经被引入诸多领域, 包括技术创新、组织学习、组织行为、领导行为、人力资源、质量 管理、战略管理、联盟关系等。本文则只关注战略联盟的活动，简称双元战略联盟。目前，有 关于双元的研究大多是关注企业 (组织内) 的单一层面的双元, 战略联盟 (组织间) 的双元 的研究比较少。

\subsection{1双元组织}

March[1]最早提出了组织双元性的概念, 随后的研究丰富和发展了组织双元性的内涵。在 March 等学者研究的基础上, Benner 和 Tushman[2]进一步提出探索性创新和挖掘性创新是两 种重要的创新类型和方式。0'Rei11y 和 Tushman[3]提出, 组织双元性可以通过间断连续、不 同部门的同时开展以及个人在时间安排上得到体现。Gupta[4]从时间和组织层级的角度对组 织双元性进行了探讨, 而 $\mathrm{CaO}$ [5]等从竞争优势的角度提出组织双元是指既能充分利用现有优 势又能抓住新机会的能力, 此能力对提升企业竞争力和绩效有益。

\subsection{2战略联盟中的双元学习}

关于战略联盟中的双元学习, 已有研究在单一组织层面尤其是 March[1]研究的基础上, 对相 关概念的内涵进行了探索. 例如, Lavie 和 Rosenkopf[6]对联盟形成决策中探索性学习与应 用性学习的平衡问题进行了探讨, 对联盟形成时的双元学习进行了界定, 但对联盟建立后的 
双元学习缺乏必要的阐述。Gupta[4]等从联盟运行过程中双元学习实现的类型出发, 提出探 索性学习和应用性学习的双元均衡和间断均衡概念, 丰富了战略联盟中双元学习的概念内涵 研究。然而, 联盟中的探索性学习和应用性学习并非完全独立的, 它们之间存在一种传递性。 较早的研究认为, 探索性联盟能够促进新产品的发展, 进而推动应用性联盟的建立, 实现在 产品市场上的成功, Russo 和 Vurro[7]也验证了组织内的探索性学习能够与组织间的应用性 学习相协调、组织外的应用性学习能够与组织内的探索性学习相协调, 进而提升联盟绩效。

2.2.3战略联盟中的双元创新

在 March 等研究的基础上, Benner 和 Tushman[2]从知识与技术轨道以及顾客与市场细分的偏 离程度解释并区分了两类创新的内涵, 验证了应用式创新与探索式创新存在排斥作用。随着 研究的不断深入, 学者们开始对这种绝对的二分法提出了质疑。Tushman 和 0' Rei11y[3]认 为，企业应同时具有在成熟市场上竞争的能力和在新兴市场上探索新产品和服务的能力。组 织双元创新对企业的正向作用已有众多研究, 而对战略联盟双元创新的讨论仍处于起步阶 段。Im 和 Rai[8]指出, 探索和应用活动对于长期组织间关系的成功同等重要。基于 He 和 Wong 以及 Jansen 等学者的研究, 我们可以将联盟双元创新定义为: 联盟同时追求高水平的应用 创新和探索创新，并使二者的水平相当。

2.2.4情境双元

目前学术界对双元的定义还存在较大的分歧, 主要有两种不同的观点: 一种侧重于从结构的 角度定义双元, 称之为 “结构双元” ; 另一种侧重于从情境的角度定义双元，称之为 “情境双 元”。Gibson 和 Birkinshaw[9]认为不应忽略 “结构双元” 所面临的高协调成本。因而，提 出了 “情境双元” 的概念, 并识别出张力、纪律、支持及信任是构建情境双元的系统要素, 如 信任, 支持, 绩效管理来培养员工的双元性思维能力, 认为情境型双元是比结构型双元更好 地实现组织双元能力的一种设计。Pate1 P. C [10]等人在此基础上提出, 通过高绩效工作系 统 (HPWS) 进一步研究获得协同性能力和适应性能力, 从而提升组织双元性能力的作用机制。

\section{3. 双元战略联盟内涵解析}

我们可以将双元战略联盟简单定义为 “同时追求应用性和探索性活动的企业联合组织” ，但 是 “同时” 所蕴含的探索和应用活动的关系是什么? 是竞争的? 还是互补的? 早期的研究提 出的探索和应用是竞争性活动的假设。基于竞争性假设可推测, 联盟组织实现双元的方式是 差异化, 即建立不同的管理系统形成结构双元, 分别培养探索性活动和应用性活动所需要的 员工技能等。但是随着不断发展，有学者研究指出探索和应用是互补性的，强调探索和应用 活动可以产生互补的知识、信息和资源。因此, 一些学者提出实现组织双元的机制是行为整 合, 即建立行为情境、知识情境或心理情境形成情境双元, 鼓励员工进行双元活动。而最新 的研究提出的探索和应用是悖论性活动的假设, 即两者之间的竞争性和互补性并不是静止不 变的, 而是动态转化的。基于这一假设, 一些学者主张融合差异化和行为整合来达成领导双 元以实现组织双元。本文将从互补的假设视角一一情境双元来解析双元战略联盟的内涵。

2.3. 概念分析

情境双元性可以被看作是一种 “元级” 能力（一致性和调整性），它能够将单位内部所有的 功能和层级联系起来。双元战略联盟的情境双元是指构建均衡的联盟组织间情境, 促使各企 业合理地处理注重效率的应用活动与侧重变革的探索活动之间的冲突性需求。本文根据 Gibson 和 Birkinshaw[9]正式提出的概念, 认为情境双元下的双元战略联盟是指在整个业务 单位范围内同时进行探索式联盟和应用式联盟的联盟组织。情境双元的实现体现在两个层面。 第一, 情境双元强调通过参与联盟的企业的双元行为来整合探索活动和应用活动, 允许企业 在两种活动上分配不同的资源、时间和努力程度。第二, 构建合适的组织情境, 利用精心设 计的文化、制度和流程来激励员工在日常工作任务中整合、处理好这些具有相对性质的活动, 促进企业双元行为向联盟双元行为的跃升。 
情境双元是与结构双元不同的双元模式，将具有双元情境的企业进行联盟，就实现了联盟情 境双元，例：有 $\mathrm{ABCD}$ 等情境双元企业进行联盟合作，比如 $\mathrm{A}$ 企业和 $\mathrm{B}$ 企业就领域 $\mathrm{p}$ 探索联盟， $\mathrm{B}$ 企业和 $\mathrm{C}$ 企业就 $\mathrm{q}$ 领域应用联盟, $\mathrm{C}$ 企业和 $\mathrm{D}$ 企业就 $\mathrm{m}$ 领域探索联盟, $\mathrm{D}$ 企业和 $\mathrm{A}$ 企业就 $\mathrm{n}$ 领域应用联盟，四个企业内部具有情境双元（或者部分企业结构双元），这样就把单个企业 双元活动扩展到多个企业了, 形成了双元战略联盟, 这种模式降低了平衡策略实施中某些冲 突的激烈程度，其面临的资源争夺、组织惯例冲突等战略悖论就会相应地减少。

2.4. 维度分析

换个视角来看, 双元战略联盟具有情境双元也可以看为由两个存在明显差异性, 但是又有一 定联系的两个维度：一种定义为双元性的平衡维度，另外一种定义为双元性的联合维度。这 两个维度通过完全不同的方式对联盟企业的绩效产生作用。平衡维度可以定义为探索和应用 之间的绝对差，也就是相对大小，联合维度可以定义为探索和应用之间的交互结果，也就是 绝对水平。平衡维度衡量着探索和应用对企业联盟占用的资源比例，联合维度可以衡量联盟 内部在实现探索和应用双元战略之后所实现的绩效[11]。

3.2 .1 战略联盟中的情境双元性创新

把上述研究成果扩展到情境双元性战略联盟创新领域：情境双元性创新的平衡维度是指探索 式创新和应用式创新高水平的平衡, 或者说是两者数量更加紧密地吻合, 其有助于联盟绩效 的大幅提高且可以有效控制绩效风险。基于这个逻辑, 在情境双元性创新的平衡维度下，我 们不难发现, 如果不能平衡应用和探索活动, 联盟会面临能力过时的风险或遭受不能获利的 危机。相反，如果能在两类创新之间适当平衡，可以促使联盟可以避免上述风险和危机。

联合维度的核心含义是探索和应用过程之间的竞争性并非不可避免。就这个问题，根据 Gupta[4]指出的 “探索和应用也会发生在互补的领域, 从而没有发生资源的竞争” 这一理论, 我们可以提出在联盟中探索和应用活动能够相互支持, 从而两类活动相互作用的效果都得到 提高的观点。就应用活动对探索活动的正向影响而言，高水平的应用活动常常可以提高联盟 探索新知识，支持新产品、新市场的效果。大量重复使用已有知识和资源，管理者可以更好 的掌握联盟内部资源的情况, 更好的理解已有知识和资源的功能。这种深刻理解可以使联盟 能够重新联合内部的既有的资源和知识, 重组与新市场和新产品发现有关的能力。总而言之, 联盟的知识和资源可以在两类活动之间进行流通, 探索和应用活动常常能相互补充来创造更 高的联盟绩效。

根据以上分析, 我们可以推断当联盟维持较高水平的探索和应用的平衡时, 两者高效率的联 合将对联盟绩效有更正向的影响。联盟追求探索式创新和应用式创新的联合是因为其提供了 更大的潜力来开发和使用探索和应用活动之间的知识和资源，因而给联盟应用新、旧知识和 资源坚实的基础。进一步推论，我们可以提出高水平的探索和应用的平衡将发挥两者的潜力。 这是因为，如果联盟应用活动远低于探索活动，其吸收能力不足，新获取的知识和资源不能 充分地被既有知识和资源吸收和处理。因此, 新吸收的知识和资源也就不能被互补的应用活 动有效使用。如果联盟的探索活动远低于应用活动, 只有较少的新知识和资源进入联盟, 这 将限制联盟的应用规模。在两种情形里, 探索和应用活动之间的交互作用都是有限的, 因此, 联合作用的效果也是有限的。相反, 如果联盟具有探索和应用活动的高度平衡, 既有知识和 资源可以被充分地使用于获取新知识和资源, 同时新知识和资源也可以被充分地整合到既有 知识和资源中去。所以, 我们认为高水平的平衡和高效率的联合对企业绩效有正向的交互作 用效果。

\section{2 .2 战略联盟中的情境双元性学习}

这两个维度在组织双元学习上研究的也很多, 我们试图分析双元战略联盟的情境双元学习。 双元学习的平衡维度表示企业的探索式学习和应用式学习的平衡程度和水平。王凤涁等[12] 采用有机平衡观将双元性界定为高能平衡、低能平衡和不平衡 3 种状态, 发现双元学习平衡 对企业创新绩效有明显提升效应, 并且高能双元学习平衡更有利于提高企业创新绩效。根据 这一理论, 我们可以进一步推断出, 高能双元学习平衡也更有利于联盟创新绩效的提高。因 
此，联盟应积极推动企业间的双元学习并在两种学习方式间平衡地分配资源，充分发挥双元 学习的平衡效应对联盟成长的重要作用。

双元学习的联合维度表示企业的探索式学习和应用式学习相互补充、相互促进的程度。 Atuahene-Gima 和 Murray[13]认为双元学习的联合效应与企业的创新绩效负相关, 但是更多 研究发现二者是正相关的。Colbert、Fang、Colombo 和Wei 等多位学者都认为探索式学 习和应用式学习的互补能够提高企业对外部知识的吸收能力, 它们相互作用所产生的复杂能 力, 能超越单独的任何一种活动, 能够为企业的竞争优势提供额外来源的能力, 对创新和绩 效具有正向作用。近年来, 学术界对探索式学习和应用式学习关系的研究重心经历了从排斥 到互补的转换。越来越多的学者关注二者的互补性, 认为这两种学习之间存在回归和共同演 化的关系。因此, 我们可以进一步推论, 探索式学习和应用式学习二者交互作用, 能够促进 联盟绩效的提高。具体而言, 由于探索式学习的高成本性, 决定联盟组织间的探索性活动需 要应用性活动的支持, 对现有技术领域知识的学习与挖掘, 可以促进联盟更有效地探索全新 的领域, 从而实现联盟企业间技术创新水平的不断提高, 所以对现有知识的挖掘和对新的互 补知识的探索是共同发展的。探索式学习能增加联盟企业之间的知识存量, 从而促进应用式 学习与创新更好的发展, 而应用式学习能为探索式学习与创新提供低成本的支撑。因此, 联 盟应充分发挥情境双元下双元学习对联盟成长和绩效的交互效应, 而不应将探索式和应用式 两种学习方式割裂开来。

\section{4. 案例分析一一以 “埃森哲-华为-SAP战略联盟” 为例}

首先, 我们不妨来看一组数据：2010 年华为首次进入《财富》世界 500 强, 排名第 397 名, 2017 年，华为排名《财富》世界 500 强第 87 位； 2014 年埃森哲首次进入《财富》世界 500 强，排名第 405 名， 2017 年，埃森哲排名《财富》世界 500 强第 305 位； 2016 年 SAP 第一 次进入《财富》世界 500 强, 排名第 462 名, 2017 年 SAP 公司排名《财富》世界 500 强第 443 位。

华为公司是全球领先的信息与通信技术 (ICT) 解决方案供应商, 专注于 ICT 领域, 在电信运 营商、企业、终端和云计算等领域构筑了端到端的解决方案优势，为运营商客户、企业客户 和消费者提供有竞争力的 ICT 解决方案、产品和服务。埃森哲公司是全球最大的管理咨询、 信息技术和业务流程外包的跨国公司。埃森哲通过企业策略、业务流程、信息技术和人员组 织的紧密结合, 帮助客户实现具有深远意义的变革, 提高客户的绩效水平, 并以出众的领导 能力、成功决心、专业服务和质量承诺在客户中享有盛誉。SAP 公司专注于企业应用软件的 研发和推广, 是全球最大的企业管理和协同化商务解决方案供应商、全球第三大独立软件供 应商。查阅相关资料后，我们不难发现，三家公司的迅速发展与其所在的双元战略联盟的成 功发展密切相关。我们不妨来探究一下这个实现多赢的战略联盟—— “埃森哲-华为-SAP 战 略联盟”。

“埃森哲-华为-SAP 战略联盟”主要的营业项目就是依托优质的服务和一流的技术与设备, 为 来自不同国家、不同行业的客户提供 SAP 企业云解决方案, 共同助力企业组织通过数字化转 型成为高敏捷型企业。

自 2012 年华为与 SAP 建立全球技术联盟合作伙伴关系以来， “华为-SAP 战略联盟”一直紧 密地聚合技术和市场能力, 整合各自优势产品, 并在多个领域开展前瞻性研究, 共同致力为 市场打造全新解决方案。目前已经在 ICT 基础设施、云计算和 “工业 4.0” 等领域联合推出 了多项创新成果, 并成功为全球数百上千家大中型企业, 提供了服务和解决方案。无疑, “华 为-SAP 战略联盟” 是一个在 ICT 基础设施、云计算和 “工业 4.0” 等领域实现先探索联盟再 应用联盟的双元战略联盟组织。

2014 年 10 月 16 日，华为和埃森哲正式签署战略联盟协议，共同面向电信运营商和企业信息 与通信技术 (ICT) 两大领域的客户需求, 开发并推广创新解决方案。“华为-埃森哲战略联 盟” 充分利用华为领先的软硬件产品和丰富的解决方案组合, 以及埃森哲在战略、咨询、数 
字技术和运营领域的能力与经验，为全球客户提供基于埃森哲 “基础设施即服务” 解决方案 和华为云计算基础设施而预先设计的一体化企业云解决方案。无疑，“华为一埃森哲战略联盟” 是一个在电信运营商和企业信息与通信技术（ICT）两大领域实现应用探索的双元联盟组织。 埃森哲与 SAP 的合作超过 30 年，建立了持久的伙伴关系。作为战略联盟， “埃森哲-SAP 战 略联盟” 以开发合适的企业解决方案以满足客户不同的需求帮助他们解决具体问题为重点。 其在解决方案产品上功能互补，在推向市场的众多业务上相互合作，包括财务服务、客户关 系管理及 SAP NetWeaver 等领域。作为埃森哲和 SAP 联盟服务的核心，迄今为止最大规模的 SAP 部署是由埃森哲管理的。埃森哲拥有 150 多位拥有 SAP 应用构建经验的咨询专家, 是唯 一拥有此资源的系统集成商。无疑，“埃森哲-SAP 战略联盟” 是一个在财务服务、客户关系 管理及 SAP NetWeaver 等领域实现了探索应用的双元联盟组织。

换个视角, 我们从维度分析的角度切入, 以战略联盟中的情境双元性创新为例, 在 “埃森哲华为-SAP 战略联盟” 中, 埃森哲作为全球一流的咨询公司，则以咨询服务、落地实施和在发 达国家市场的稳步发展见长, 而华为在 ICT 基础设施上拥有丰富的专业经验, 以及多年深耕 新兴市场积累了大量开拓市场经验, SAP 则专注于企业级软件产品, 三者在各领域里形成了 互补的产业分工, 高度平衡了探索和应用活动。不仅如此, SAP 已经与华为在中国深圳建立 了一个联合创新中心。埃森哲有 13,000 名熟悉 SAP 的专业人才, 他们对 SAP 各行业的产品都 很熟悉。此外埃森哲在全世界有 40 多个交付中心他们能提供 SAP 技术及外包服务。这些联合 团队组织追求同时追求探索式创新和应用式创新，为联盟整体提供了更大的潜力来开发和使 用探索和应用活动之间的知识和资源，因而给联盟应用新、旧知识和资源坚实的基础。

因此，“埃森哲-华为-SAP 战略联盟” 具有探索和应用活动的高度平衡，保证了既有知识和 资源可以被充分地使用于获取新知识和资源, 同时也使新知识和资源可以被充分地整合到既 有知识和资源中去，实现了战略联盟下情境双元下的双元创新的平衡与联合。

综合上述分析，我们可以发现 “埃森哲-华为-SAP 战略联盟” 是一个基于不同领域不同情境 下双元战略联盟。

\section{5. 结语}

综合上文，双元战略联盟是两个或两个以上的企业基于共同的愿景和战略目标，在不同领域， 为了维持联盟以及企业自身的竞争优势, 而形成的能够高水平协同运用情境双元性的平衡和 联合维度来协调双元性矛盾, 达成学习与创新层次上的探索与应用的双元平衡的既松散又紧 密的互补型的组织。

本文在情境双元的视角上, 结合战略联盟和组织双元战略延伸论证, 从概念以及维度两方面 分析了双元战略联盟的内涵, 建立行为情境、知识情境或心理情境来鼓励员工进行双元活动, 在不同的领域用互补的视角建立双元战略联盟，促进双元战略联盟的探索与应用活动的平衡 与联合，促进双元战略联盟的成功实施。最后本文用 “埃森哲-华为-SAP 战略联盟” 的例子 论证了双元战略联盟的内涵。通过本文的分析探索, 希望能够给当前陷入塩尬境地的战略联 盟组织带来新的发展方向的启发。

\section{Acknowledgements}

课题基金: 供给侧结构性改革背景下双元创新驱动的制造业服务化问题研究.

Study on the service innovation of manufacturing driven by dual innovation under the background of supply-side structural reform. 


\section{References}

[1]. March. Exploration and exploitation in organizational learning[J]. Organization Science,1991, 2(1): 71-87.

[2]. Benner, M. J, \& Tushman, M. L. Exploitation, exploration and process management: The productivity dilemma revisited. Academy 0f Management Review, 2003, 28(2): 238-256.

[3]. Tushman M L, O' Reilly C A. The ambidextrous organization: Managing evolutionary and revolutionary change[J]. California Management Review, 1996, 38(4): 1-23.

[4]. Gupta A. K, Smith G K, Shalley C E. The interplay between exploration and exploitation [J], Academy of Management Journal, 2006, 49( 4) : 693-706.

[5]. Cao Q, Gedajlovic E, Zhang H. Unpacking organizational ambidexterity: dimensions, contingencies and synergistic effects[J]. Organization Science, 2009, 20 (4) :781-796.

[6]. Lavie D, Rosenkopf L. Balancing exploration and exploitation in alliance formation[J]. Academy of management Journal, 2006, 49(4): 797-818.

[7]. Russo A, Vurro C. Cross-boundary ambidexterity: Balancing exploration and exploitation in the fuel cell industry[J]. European Management Review, 2010, 7(1): 30-45.

[8]. Im G, Pail A. Knowledge Sharing Ambidexterity in Long - term Inter - organizational Relationships[J]. Management Science, 2008, 54 (7): 1281-1296.

[9]. Gibson C B, Birkinshaw J. . The antecedents, consequences and mediating role of organizational ambidexterity[J].Academy of Management Journal, 2004, 47(4): 209-226

[10]. Patel P C, Messersmith J G, Lepak D P. Walking the tightrope: An assessment of the relationship between high performance work systems and organizational ambidexterity[J]. Academy of Management Journa1, 2013, 56 ( 5) : 1420-1442

[11]. Li C R, Disentangling the effect of exploratory learning and exploitative learning in product innovation process[J]. Canadian Journal of Administrative sciences(John Wiley and Sons, Inc. ), 2013, 30(2): 101-114.

[12]. Wang Fengbin, Chen Jianxun, Yang Yang. An Analysis of the Effect of the Explorative and Exploitative Innovations and their Balance. Management World, 2012, (3): 96-112.

[13]. Atuahene-Gima K. , Murray J. Y. Exploratory and Exploitative Learning in New Product Development: A Social Capital Perspective on New Technology Ventures in China[J]. Journal of International Marketing, 2007,15( 2) : 1-29. 